\title{
Control System of Manipulator Based on Steady State Visual Evoked Potential
}

\author{
Pengfei Song \\ 1 Tianjin Key Laboratory of Information Sensing and Intelligent Control, Tianjin University of Technology and Education No.1310,Dagu \\ South Road, Hexi District, Tianjin 300222, China
}

\begin{abstract}
The steady state visual evoked potential is used as the input signal of manipulator control system, then a manipulator control system based on steady state visual evoked potential has been designed and the control of 6 kinds of motion of the manipulator with the EEG signals has been achieved. The system first detects the components of steady state visual evoked potential in electroencephalogram signals, and then converts them to the control commands of the external manipulator after feature extraction and pattern recognition. The result that the recognition accuracy of the control system designed in this paper is more than $73 \%$ has been validated by a series of experiments. Therefore, this system provides a new method for the control of machine behaviors.
\end{abstract}

Keywords: Manipulator, Steady State Visual Evoked Potential, Signal processing.

\section{Introduction}

Brain computer interface (BCI) refers to the direct control channel established between human brain and computer or other electronic devices, which does not depend on the normal physiological output of the brain. The main purpose of the research and development of BCI technology is to design a control device based on the electroencephalogram (EEC) to help patients with severe physical disability and abnormal communication in communication and control with the external environment. The traditional human-computer interaction is realized by sound, buttons, etc., but some old people and people with disabilities do not have the ability of language expression or limb operation because of various reasons, so the BCI technology provides a new way for them to communicate with the outside world[1].

The EEC signals can be used for brain-computer interface are P300, visual evoked potential, cortical slow potential, spontaneous EEC and so on. Among them, the visual evoked potential has a relatively stable waveform characteristics and a unique relationship with the visual stimulus, so it is easier to detect and it has little relationship with the self-control ability of the subjects making it suitable to act as the input signal of brain-computer interface[2-3].

The author designs a manipulator control system based on the steady state visual evoked potential, and then the EEC signals can conduct the real-time control of the 6 kinds of actions, namely rise, fall, left, right, clamp and release. By detecting the components of steady state visual evoked potential in EEC signals, the system conduct feature extractions and pattern recognitions of evoked potentials by using signal processing procedures such as wavelet de-noising and Fourier transform and then convert it to the control command for external manipulator. Experiments show that the control system can accomplish the control of the manipulator so the realization of the system is a new exploration for human on the control of the machine.

\section{System Composition}

As shown in Figure 1, the manipulator control system based on the steady state visual evoked potential includes four segments: steady state visual evoked, signal collection, signal processing and output control[4].

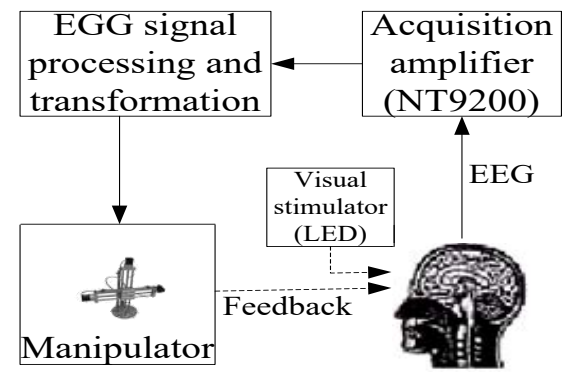

Figure 1: Control system block diagram

The system hardware includes visual stimulator, digital electroencephalogram instrument and manipulator. The visual stimulator composes of six white LED light-emitting blocks controlled by ATmega16 singlechip. The size of the light-emitting block is $2 \mathrm{~cm} \times 2 \mathrm{~cm}$ and the separation distance of each light-emitting block is $2.5 \mathrm{~cm}$. In addition, the stimulus frequency of each light-emitting block is adjustable between 7 $\mathrm{HZ}$ to $20 \mathrm{HZ}$ with the frequency error less than $0.1 \mathrm{HZ}$. The digital electroencephalogram instrument uses NT9200 series electroencephalogram instrument produced by Beijing Zhong Ke Xin Tuo instrument Co., Ltd. The manipulator part includes one pivot joints and two movable parts that can be moved around and down. The clamp can drive it to clamp and release and the structure of the manipulator used in the system is shown in Figure 2. The stimulator is mainly used to induce steady-state visual evoked potentials and the digital electroencephalogram instrument is used to collect the scalp EEG signal which will be transmitted to the computer for processing. Then the processing result will be converted to the control command of the manipulator and the real-time control of the manipulator will be achieved by communication between the wireless network and the manipulator using the computer serial port. In this process, wavelet transform and the 


\section{International Journal of Science and Research (IJSR) \\ ISSN (Online): 2319-7064}

Index Copernicus Value (2013): 6.14 | Impact Factor (2014): 5.611

Fourier transform and other methods are used to implement the EEC signal processing.

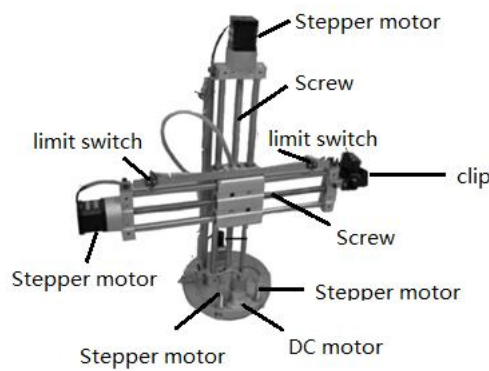

Figure 2: Schematic diagram of manipulator

\section{Characteristic Detection of Potential}

Generally speaking, the amplitude of visual evoked potentials of adults is about several microvolts to tens of microvolts which is weak and usually submerged in strong background noise. Therefore, the EEC signals collected from the scalp need to be pretreated firstly to remove background noise mixing in the EEC signals and to improve the signal to noise ratio, and then the feature extraction of evoked potentials can be conducted. In this paper, we use wavelet transform to remove background noise and conduct feature extraction of steady state visual evoked potential using Fourier transform.

\subsection{Denoising method based on The Wavelet Transform}

Wavelet transform is a analysis method on time and scale and it has the ability to indicate the local characteristic of signal with the characteristics of multi-resolution analysis, so wavelet transform is widely used in fields of signal analysis[5].

If the Fourier transform $\psi(w)$ of the function of $\psi(t) \in L^{2}(R)$ satisfies the condition:

$$
C_{\psi}=\int_{R} \frac{|\psi(\omega)|^{2}}{\omega} d \omega<\infty
$$

Then $\psi(t)$ is a basic wavelet or wavelet function. And

$$
W T_{f}\left(a^{j}, k \tau_{0}\right)=\int x(t) \psi_{a^{j}, k \tau_{0}}^{*} d t
$$

is discrete wavelet coefficient generated by the mother wavelet function $\psi_{a^{j}, k \tau_{0}}$ and $W T_{f}\left(a^{j}, k \tau_{0}\right)$ is discrete wavelet transform of the function.

Discrete stationary wavelet transform is a kind of non orthogonal wavelet transform with the characteristics of shift invariance, so it is more suitable for signal denoising, especially for the removal of correlation noise compared with the traditional discrete orthogonal wavelet transform. In addition, the the number of wavelet coefficients of each level is eaqual with the number of original signal after it is decomposed, so it can be ensured that there are enough coefficients in all sub bands of each layer to obtain the optimal threshold in the process of wavelet denoising which can decrease the Gibbs inkstone appearing after the signal reconstruction. So the author mainly uses the discrete stationary wavelet transform to remove all kinds of noises mixing in the EEC signal. The steps of denoising can be roughly divided into the following steps: (1) Selection of wavelet basis function and the Sym8 wavelet is finally adopted after comparison. (2) Determination on decomposition leve. The signal sampling frequency is $100 \mathrm{~Hz}$ and the signal is decomposed into 3 layers, then the frequency ranges of each component after the decomposition are roughly: $d_{1}: 25-50 \mathrm{~Hz}$, $d_{2}: 12.5-25 \mathrm{~Hz}, d_{3}: 6.25-12.5 \mathrm{~Hz}, a_{3}: 0-6.25 \mathrm{~Hz}$; (3) threshold quantization of coefficients at all levels after the decomposition. The main components of the noise are the baseline drift and eye movement disturbance which are mainly concentrated in the low frequency, so this step is mainly used to remove the components at low frequency.(4) The reconstruction of the signal is carried out using the wavelet coefficients at different levels after the quantization, and the majority of the coefficients of the $\mathrm{d} 2 \sim \mathrm{d} 3$ components are mainly used in this step.

\subsection{Feature Extraction}

Fourier transform is a basic analysis method in frequency domain of signal. We often should know the local frequency of the signal and the time information of this frequency when dealing with non-stationary signals. Because the standard Fourier transform only has local analysis ability in the frequency domain, but has no local analysis ability in the time domain, so Gabor introduced the short-time Fourier transform in 1946. The basic thought of short-time Fourier transform is to make the signal divided into many small time intervals analysed with Fourier transform in order to determine the frequency during this time interval.

The energy of the steady state visual evoked potentials which is evoked by the specific stimulation frequency is mainly concentrated at the specific frequency, so the algorithm of the feature extraction of EEC signals is simplified. The author uses short-time Fourier transform for feature extraction taking into account the needs of real-time system speed.In the short-time Fourier transform, a short-time Fourier transform is conducted for each $2 \mathrm{~S}$ data and then the stimulus frequency at which the maximum amplitude appears can be decided. And the stimulus frequency is considered to represent the control commands which the subjects want to issue when the same result appears for three consecutive times.

\section{Performance Analysis of Motion Control System}

The performance test of the manipulator control system based on steady state visual evoked potential was performed in a quiet room and there were six subjects participating in the test whose average age was 23 years with normal vision or corrected visual acuity. In this experiment, the subjects wearing electrode caps were required to sit at a place about $50 \mathrm{~cm}$ away from the stimulator and look at some flash block of the stimulator horizontally with a relaxed state. Electrodes adopted to the system standard electrode placement method of international $10-20$, which were located in the occipital

\section{Volume 4 Issue 12, December 2015}




\section{International Journal of Science and Research (IJSR) \\ ISSN (Online): 2319-7064}

Index Copernicus Value (2013): 6.14 | Impact Factor (2014): 5.611

region named $\mathrm{O} 1$ and $\mathrm{O} 2$, and the reference electrode was two lobes with $\mathrm{CZ}$ electrode earthed and a signal sampling frequency of $100 \mathrm{~Hz}$. In addition, the frequency of the visual stimulation was set to $7,9,11,14,16,18 \mathrm{HZ}$.

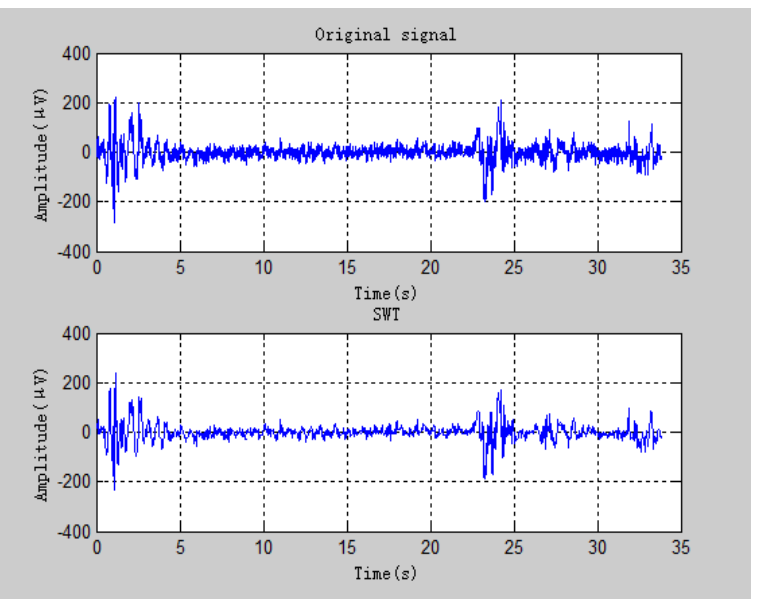

Figure 3: The original signal and the signal after SWT

Analyses were made taking the experimental results of a certain subject as an example. A section of the primary EEC signal produced when the subject staring at the target of $14 \mathrm{~Hz}$ was collected, and the signal is shown in Figure 3 after the pretreatment with wavelet transformation.

From Figure 3, we can see that, the EEC signals after de-noising basically do not include the baseline drift and eye movement interference in the signal and the signal shape is generally saved with better de-noising effect. After de-noised signal treated with a short-time Fourier transform, the spectrums at stimulus frequency are shown in Figure 4.

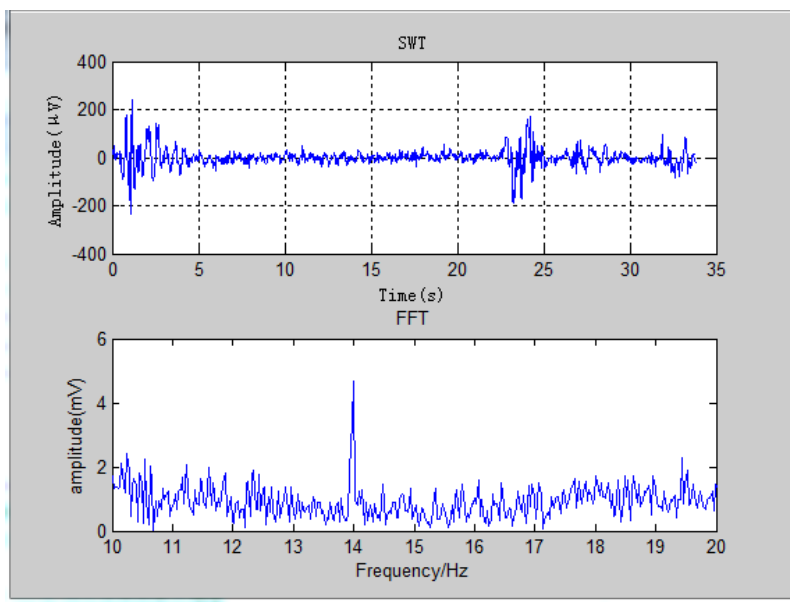

Figure 4: The signal after FFT

Figure 4 shows that the feature of visual evoked potential extracted from the subject is obvious. The statistic result is shown in Table 1 about the accuracy rate of 6 kinds of mechanical hand movements being controlled successfully by all the six subjects participating in the experiment.
Table 1: Experimental result

\begin{tabular}{|c|c|c|c|}
\hline Subjects & $\begin{array}{c}\text { Command } \\
\text { numbers }\end{array}$ & $\begin{array}{c}\text { Number of } \\
\text { successes }\end{array}$ & $\begin{array}{c}\text { Success } \\
\text { rate } / \%\end{array}$ \\
\hline ZZ & 30 & 22 & $73.33 \%$ \\
\hline SHQ & 30 & 26 & $86.67 \%$ \\
\hline WFF & 30 & 25 & $83.33 \%$ \\
\hline CYX & 30 & 25 & $83.33 \%$ \\
\hline LW & 30 & 27 & $90 \%$ \\
\hline ZK & 30 & 24 & $80 \%$ \\
\hline
\end{tabular}

From table we can see that, the average accuracy rate of the five subjects is $82.78 \%$ and the accuracy rate of the worst performer is also more than $73.33 \%$, so the identification results can complete better real-time control on the manipulator.

\section{Conclusion}

The real-time control system of multi DOF manipulator based on the steady state visual evoked potential is designed, and the control of 6 kinds of motions of the manipulators is realized. By extracting the components of steady-state visual evoked potential in the EEC signal, the feature extraction and pattern recognition of the signal are conducted with wavelet denoising and short-time Fourier transform and other signal processing methods, and then the signal will be converted to different control commands for an external manipulator. Experiments show that the control system can accomplish the control of the manipulator, so the realization of the system provides a new way for the control of the machine.

\section{References}

[1] Xiao Dong Zhang, Rui Li, Yao Nan Li. Research on Brain Control Technology[J].Journal of Vibration, Measurement \& Diagnosis, 2014, 34(2): 205-211

[2] Jian Ming Xu, Jian Zhang, Li Yu. Design of manipulator motion control system based on ARM[J].Journal of Zhejiang University of Technology, 2014, 42(3): 243-248

[3] Wilson J J, Palaniappan R. Analogue mouse pointer control via an online steady state visual evoked potential (SSVEP) brain-computer interface[J]. Journal of neural engineering, 2011, 8(2): 025026.

[4] Fang Bo Liao. Study on localization and mapping of a mobile robot based on sensors fusion[D].BeiJing: Beijing Jiaotong University,2014.

[5] Jian Ming Xu, Jian Zhang, Li Yu. Design of manipulator motion control system based on ARM[J].Journal of Zhejiang University of Technology, 2014, 42(3): 243-248

\section{Author Profile}

Pengfei Song is reading the M.S. degrees in Tianjin University of Technology and Education . 